\title{
Human Resistin Inhibits Myogenic Differentiation and Induces Insulin Resistance in Myocytes
}

\author{
Chun Hua Sheng, ${ }^{1,2}$ Zhen Wu Du, ${ }^{1}$ Yang Song, ${ }^{1}$ Xiao Dong Wu, ${ }^{1}$ Yu Cheng Zhang, \\ Mei Wu, ${ }^{1}$ Qian Wang, ${ }^{1}$ and Gui Zhen Zhang ${ }^{1}$ \\ ${ }^{1}$ Department of Central Research, The Third Clinical College, Jilin University, Changchun, Jilin 130033, China \\ ${ }^{2}$ Cell Transplantation Center, The 208th Hospital of Chinese People's Liberation Army, \\ Changchun, Jilin 130021, China
}

Correspondence should be addressed to Gui Zhen Zhang; zhangguizhenjlu@yahoo.com

Received 21 August 2012; Revised 6 December 2012; Accepted 21 December 2012

Academic Editor: Joseph Fomusi Ndisang

Copyright (C) 2013 Chun Hua Sheng et al. This is an open access article distributed under the Creative Commons Attribution License, which permits unrestricted use, distribution, and reproduction in any medium, provided the original work is properly cited.

\begin{abstract}
This study is aimed to investigate the effect of human resistin on myocyte differentiation and insulin resistance. The human resistin eukaryotic expression vector was stable transfected into $\mathrm{C} 2 \mathrm{C} 12$ myocyte cells and was transiently transfected into COS7 cells. The effects of human resistin on cell proliferation, cell cycle, and myogenic differentiation of $\mathrm{C} 2 \mathrm{C} 12$ cells were examined. Glucose uptake assays was performed on $\mathrm{C} 2 \mathrm{C} 12$ myotubes by using $\left[{ }^{3} \mathrm{H}\right] 2$-deoxy-D-glucose. The mRNA levels of insulin receptor (IR) and glucose transporter 4 (GLUT4) were evaluated by semiquantitative RT-PCR. Results showed by the C2C12 cells transfected with human resistin gene compared with that without transfecting gene are as follows: (1) cell proliferation was significantly promoted, (2) after inducing differentiation, the myotube's diameters and expression of desmin and myoglobin decreased, and (3) glucose uptake ratio was lowered and expression of IR and GLUT4 decreased. However, there was no significant difference in the glucose uptake ratio between $\mathrm{C} 2 \mathrm{C} 12$ myotubes treated with a human resistin conditioned medium of COS7 cells and treated with control medium. These results suggest that maybe human resistin has not a direct role on insulin sensitivity of myocytes. However, maybe it impaired the insulin sensitivity of myocytes through suppressing myogenesis and stimulating proliferation of myoblasts.
\end{abstract}

\section{Introduction}

Resistin is known as an adipocyte-specific secretory factor (ADSF) that belongs to a gene family found in the inflammatory zone (FIZZ) or found in the resistin-like molecule (RELM) [1]. Mostly researches reach consensus on the effect of resistin inducing insulin resistance in rodents [1-6]. However, it is controversial in the studies evaluating resistin expression related to type 2 diabetes in humans [7-11]. It is necessary to investigate the effects of human resistin ( $h R$ ) on the target cells of insulin action. The skeletal muscle is a major tissue to take up and utilize glucose in vivo. Although it is argued that whether resistin is expressed in a skeletal muscle or not $[1,10,12,13]$, it impacts glycometabolism by endocrine, autocrine, and paracrine ways so that it is conceivable that effects of resistin might contribute to the pathogenesis of impaired insulin sensitivity, it is significant to elucidate the effect of human resistin on myocytes. However, some reports considered the activity of recombinant human resistin may be lower than endogenous resistin [14-16], so we constructed a human resistin eukaryotic expression vector. $\mathrm{C} 2 \mathrm{C} 12$ myoblast is originated from a skeletal muscle of mouse and forms myotube after inducing differentiation. COS7 cells, as the African green kidney cell line, express SV40 large T antigen and support the replication of plasmid vector which contains SV40 ori (including PcDNA3.1) or some mutant SV40 to obtain high-level expression of exogenous genes. As a high-performance transient expression of eukaryotic systems, it is a commonly used tool to study gene function. In this study, human resistin eukaryotic expression vector were stably transfected into $\mathrm{C} 2 \mathrm{C} 12$ myoblast and transiently 
transfected into COS7 cells to examine the effect of $h \mathrm{R}$ on myocytes.

\section{Materials and Methods}

2.1. Plasmids' Construction. The pGEM-T human resistin (pGEM-T-hR) clone vector was kindly provided by professor Ying Sun (King's College London, London, UK). Human resistin (hR) gene was amplified by polymerase chain reaction (PCR) from the pGEM-T$\mathrm{hR}$ clone vector. The primer sequences for hR amplification are as follow: forward primer: $5^{\prime}$-TCAGGT ACCATGGCCATGAAAGCTCTCTGTCTCCTC-3 ${ }^{\prime}$; reverse primer: $5^{\prime}$-TCGGAATTCTCAGGGCTGCACACGACAGC$3^{\prime}$. Both primers include additional leader sequences that corresponded to the KpnI and EcoRI restriction enzymes, respectively, that can direct hR gene cloning into the pcDNA3.1 plasmids (Pc-3.1) (Invitrogen). HR cDNA generated by PCR was confirmed by the gene sequencing and inserted into plasmid Pc-3.1 by gene recombination technology. The hR gene of the construct was named pcDNA-hR (Pc-hR). Pc-3.1 plasmid was used as a control vector.

2.2. Cell Culture and Differentiation Assays. C2C12 and COS7 cells (American Type Culture Collection) were cultured in Dulbecco's minimal essential medium (DMEM) containing $25 \mathrm{mM}$ glucose and 10\% fetal bovine serum (FBS) (GIBCO), respectively. $\mathrm{C} 2 \mathrm{C} 12$ cells were grown to $100 \%$ confluence with $10 \% \mathrm{FBS}$ serum and then changed to $2 \%$ horse serum. By day $10, \mathrm{C} 2 \mathrm{C} 12$ cells were fused into myotubes.

2.3. Stable Transfection in $\mathrm{C} 2 \mathrm{C} 12$ Cells. $\mathrm{C} 2 \mathrm{C} 12$ cells were transfected with Pc-hR and Pc-3.1 plasmids by using Lipofectamine 2000 reagent (Invitrogen) following the protocol. $72 \mathrm{~h}$ after transfection, the medium was changed to the selection DMEM supplemented with $800 \mu \mathrm{g} / \mathrm{mL} \mathrm{G418}$ (GIBCO). Three weeks later, the cell clones were screened and further cultured in DMEM medium containing $400 \mathrm{mg} / \mathrm{mL}$ G418.

2.4. Transient Transfection in COS7 Cells. The Pc-3.1 and PchR plasmids were transfected, respectively, into COS7 cells by using Lipofectamine 2000 reagent. $72 \mathrm{~h}$ after transfection, the medium of COS7 cells was collected, centrifuged at $500 \times \mathrm{g}$ for $5 \mathrm{~min}$, and stored at $4^{\circ} \mathrm{C}$ as conditioned medium of hR for less than a week before use.

2.5. Immunocytochemistry and Immunofluorescence Staining. The immunocytochemical detection of $\mathrm{hR}$ expression was carried out in $\mathrm{C} 2 \mathrm{C} 12$ cells as previously described [17] using mouse anti-human resistin monoclonal antibody $(\mathrm{R} \& \mathrm{D}$ Systems, Inc., 1: 50 dilution). The cells were visualized using a microscope (Olympus) and the immunoreactivity was identified as brown cytoplasmic staining in cytoplasm. Immunofluorescence staining was performed to identify the expression of desmin and myoglobin in $\mathrm{C} 2 \mathrm{C} 12$ myotubes using monoclonal mouse antibody to desmin (Sigma-Aldrich, 1:50 dilution) and myoglobin (R\&D Systems, Inc., 1:50 dilution) and goat anti-mouse Cy3-conjugated secondary antibody (R\&D Systems, Inc., 1:200 dilution). The cells were visualized under a fluorescence confocal microscopy (OLYMPUS confocal microscope FV500). The grayscale value of desmin and myoglobin expression and the myotubes diameters were measured from randomly selected microscope fields from five different wells of Pc-3.1-transfected and Pc-hRtransfected $\mathrm{C} 2 \mathrm{C} 12$ myotubes, respectively, and analyzed by image analytical system HPIAS-1000. At least five fields were selected and the diameter of 150 myotubes was measured per well.

2.6. Methyl Thiazolyl Tetrazolium (MTT) Assays to Determine Cell Growth. C2C12 cells transfected with Pc-3.1 and Pc-hR were seeded in 96-well tissue culture plates $\left(2 \times 10^{3}\right.$ cells/well $)$ respectively. At the indicated time points, the assays were performed as the manufacturer's instruction.

2.7. Cell Cycle Analyses. C2C12 cells were cultured in culture flasks. After reaching 30\% confluence, the cells were treated with DMEM without FBS for $24 \mathrm{~h}$ for synchronization. At the next day, cells were cultured with DMEM with $10 \%$ FBS. Forty-eight hours later, cells were trypsinized, fixed in $70 \%$ ethanol, and were analyzed by flow cytometry using a FACScan Flow Cytometer (Becton Dickinson, San Jose, CA, USA).

2.8. Glucose Uptake Assays. The measurements for glucose uptake were performed as described by Nakamori et al. [18]. Briefly, C2C12 myotubes (10 days after differentiation) transfected with $\mathrm{pc}-\mathrm{hR}$ and $\mathrm{pc}-3.1$ were grown in serum-free DMEM for $4 \mathrm{~h}$ and then incubated in the presence of insulin for 30 minutes at $37^{\circ} \mathrm{C}$. Transport was started by adding $1 \mu \mathrm{Ci}$ $\left[{ }^{3} \mathrm{H}\right] 2$-Deoxy-D-glucose (Amersham Pharmacia Biotech) in $1 \mathrm{~mL}$ of the Krebs-Ringer phosphate buffer for $10 \mathrm{~min}$ at $37^{\circ} \mathrm{C}$ and stopped by rapidly washing for three times with ice-cold phosphate-buffered solution (PBS). Cells were lysed in $0.4 \mathrm{~mL}$ PBS containing $0.1 \%$ Triton X-100 for $45 \mathrm{~min}$. Aliquots of the cell lysates were used for liquid scintillation counting and determination of protein content by the Bradford method. Nonspecific transport was assayed in the presence of $10 \mu \mathrm{mol} / \mathrm{L}$ cytochalasin B (Sigma-Aldrich). In addition, C2C12 myotubes (10 days after differentiation) were incubated in conditioned medium of COS7 cells transfected with pc-3.1 and pc-hR, respectively, for $24 \mathrm{~h}$. The cells were then changed to serum-free DMEM for $4 \mathrm{~h}$ and performed glucose uptake assays as above.

2.9. Semi Quantitative RT-PCR Analysis of $m R N A$ Expression. Total RNA was extracted from cells with the use of TRIzol reagent (GIBCO). RNA $(1 \mu \mathrm{g})$ was reverse transcribed using RevertAid H Minus M-MuL V reverse transcriptase (Helena Biosciences, Europe, Sunderland, UK) and random hexamers in $20 \mu \mathrm{L}$ reaction volume, according to the manufacturer's instructions. The products $(1 \mu \mathrm{L}$ of cDNA) were subjected to PCR with Ex Taq and primers. 


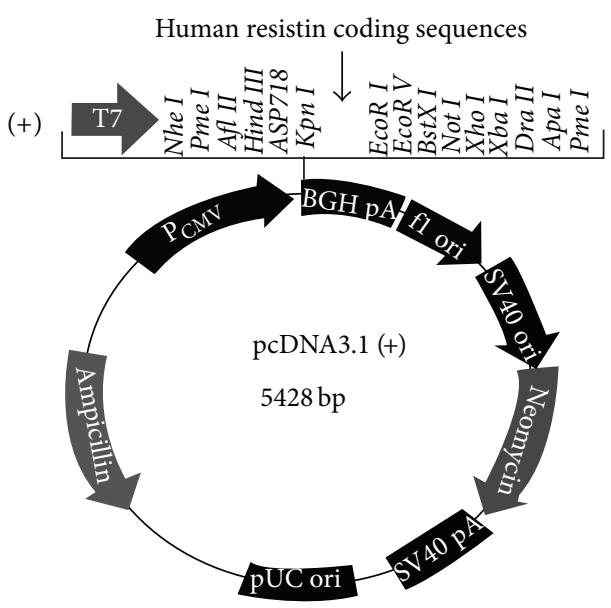

(A)

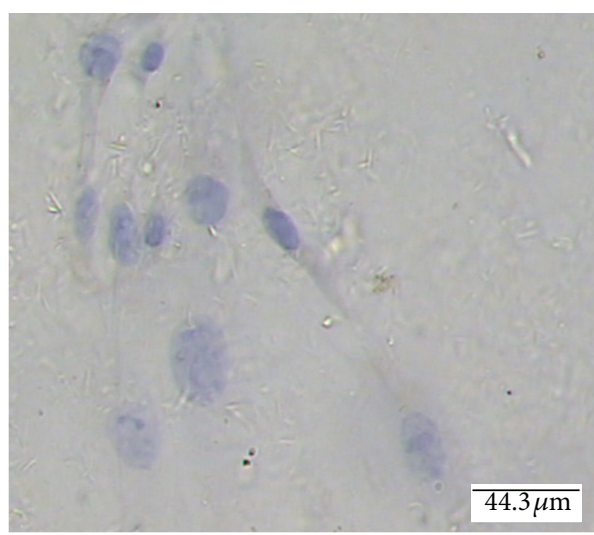

C2C12 transfected with Pc-3.1

(a)

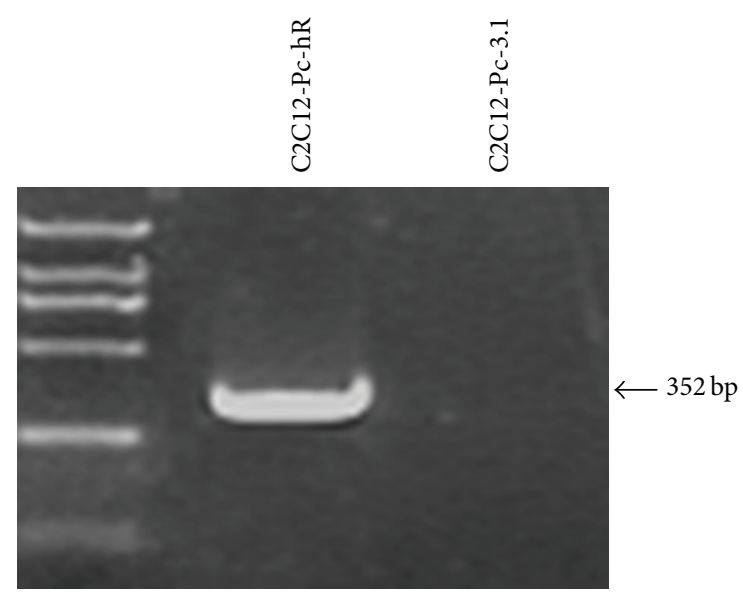

(B)

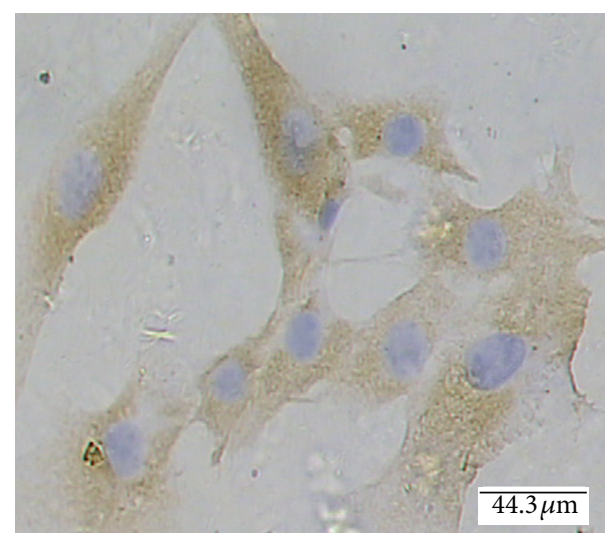

C2C12 transfected with Pc-hR

(b)

(C)

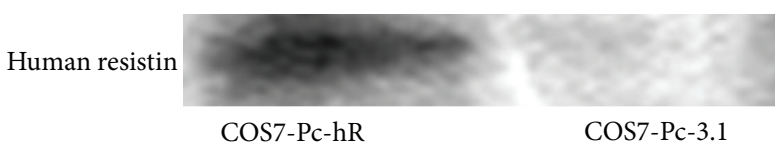

(D)

FIGURE 1: Construction of recombinant hR expression vector and transfection in cell lines. (A) A full length of hR coding sequence was inserted into PcDNA-3.1 (Pc-3.1). (B) The expression of hR mRNA was identified in C2C12 cells transfected with PcDNA-hR (Pc-hR). (C) Immunocytochemistry analysis showed hR immunoreactive protein in $\mathrm{C} 2 \mathrm{C} 12$ cells transfected with Pc-hR. (D) Western blot analysis showed expression of hR in COS7 cells transfected with Pc-hR. C2C12-Pc-3.1: C2C12 cells transfected with Pc-3.1 plasmids; C2C12-Pc-hR: C2C12 cells transfected with Pc-hR plasmids.

Primer sequences are as follow: IR: forward primer: $5^{\prime}$ ATGGACATCCGGAACAACCT- $3^{\prime}$; reverse primer: $5^{\prime}$-TTGATGACAGTGGCAGGACA-3' (the product was $493 \mathrm{bp}$ ), GLUT4: forward primer: 5'-CAACGTGGCTGGGTAGGCA- $3^{\prime}$; reverse primer: $5^{\prime}$-ACACATCAGCCCAGCCGGT$3^{\prime}$ (the product was $587 \mathrm{bp}$ ), $\beta$-actin 1: forward primer: GATGGTGGGTATGGGTCAGAAGGA; reverse primer: GCTCATTGCCGATAGTGATGACCT (the product was $632 \mathrm{bp}$ ), and $\beta$-actin 2: forward primer: $5^{\prime}$-GGGACCTGACAGACTACCT- ${ }^{\prime}$; reverse primer: $5^{\prime}$-CAGGATTCCATACCCAAG-3' (the product was $268 \mathrm{bp}$ ). The PCR products were separated by electrophoresis on agarose gel, visualized by ethidium bromide staining, and quantitated with Gel
Image Systems. The abundance of each specific mRNA was normalized on the basis of that of $\beta$-actin mRNA.

2.10. Western Blot Analysis. Cell supernatants of Cos7 cells transfected with Pc-3.1 and Pc-hR plasmids were collected and treated by western blot $72 \mathrm{~h}$ after transfection as described [19]. In brief, the proteins were subjected to SDS polyacrylamide gel electrophoresis, electroblotted onto Polyvinylidene fluoride (PVDF) membrane (Millipore), and immunodetected using mouse anti-human resistin monoclonal antibody and goat anti-mouse IgG horseradish peroxidase conjugate. 


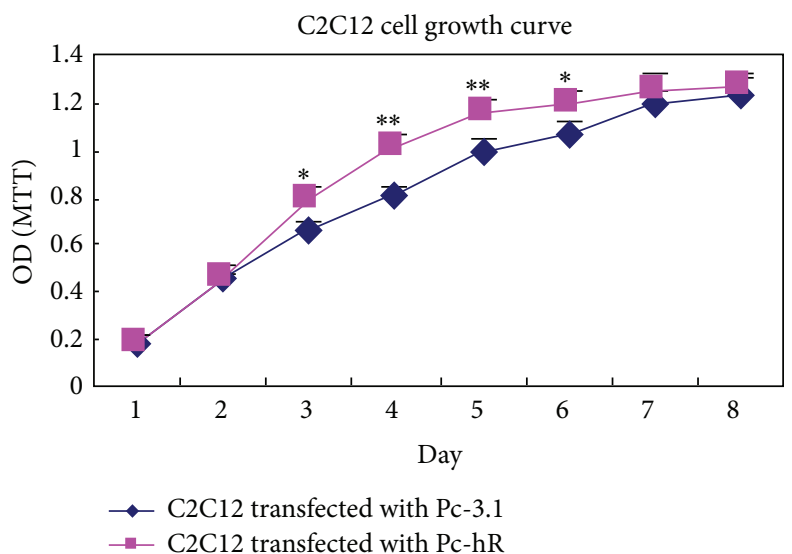

(a)

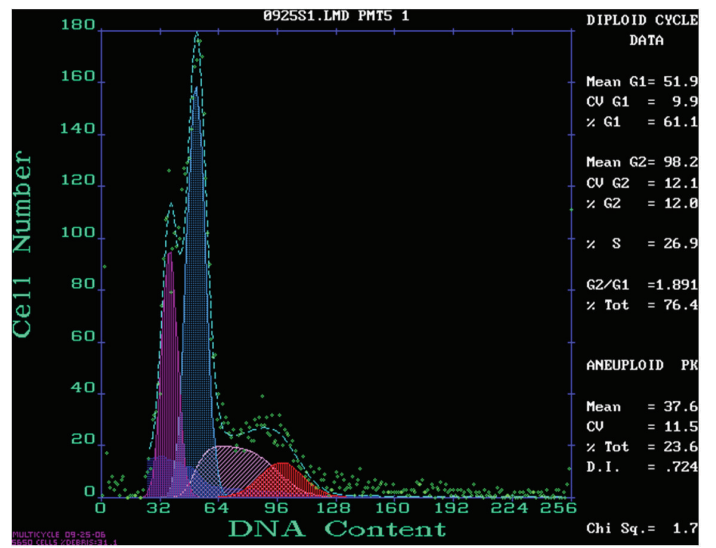

(b)

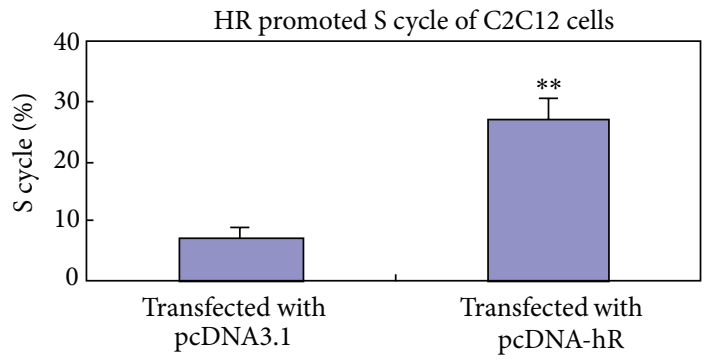

(d)

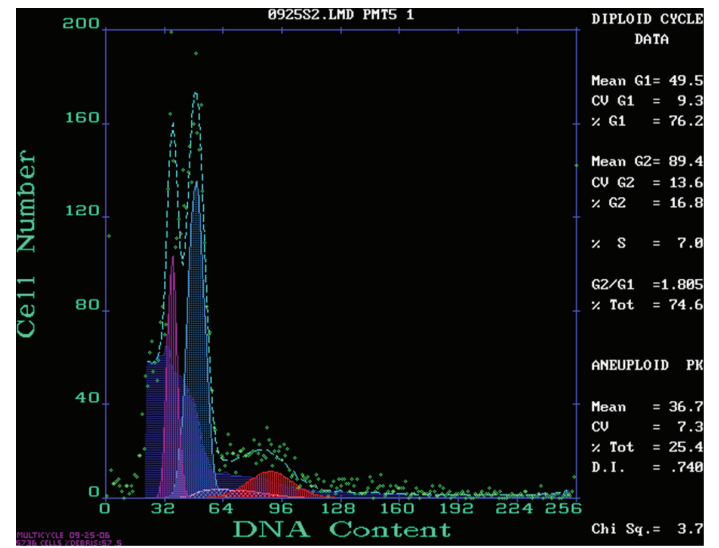

(c)

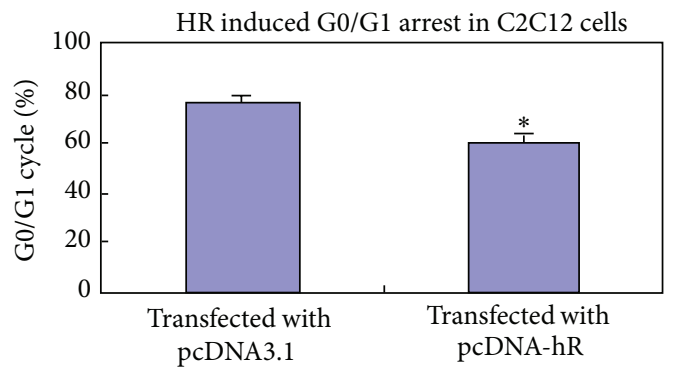

(e)

Figure 2: Effects of hR on proliferation and cell cycle of the C2C12 cells. (a) MTT showing significant increased absorbance of C2C12 cells transfected with Pc-hR compared with the control cells transfected with Pc-3.1. (b) Flow cytometry showing the cell cycle of C2C12 cells transfected with Pc-hR. (c) Flow cytometry showing cell cycle of C2C12 cells transfected with Pc-3.1. (d) Analysis of the percentages of S cycle. (e) Analysis of the percentages of G0/G1 cycle. Results are expressed as the means \pm SEM of six measurements. ${ }^{*} P<0.05$; ${ }^{* *} P<0.01$.

2.11. Statistical Analysis. Statistical analyses were carried out using SPSS (SPSS Inc. 12.0, Woking, UK) software. All qualitative data are representative of at least three independent experiments, with at least four wells per group per experiment. Quantitative data are presented as means \pm SEM and were compared with Student's $t$ test. $P<0.05$ was considered statistically significant.

\section{Results}

3.1. Construction of Recombinant Human Resistin (hR) Expression Vector and Transfection into Cell Lines. The full length of cDNA encoding hR coding sequences (327 bp) was cloned into PcDNA3.1 (Pc-3.1) vectors (Figure $1(\mathrm{~A})$ ). The recombinant plasmid PcDNA-hR (Pc-hR) and the control plasmid Pc3.1 were stably transfected into $\mathrm{C} 2 \mathrm{C} 12$ cells and transiently transfected into COS7 cells. The expression of recombinant $\mathrm{hR}$ at mRNA and protein levels in C2C12 cells were identified by RT-PCR and immunocytochemistry respectively. Expression of hR mRNA and protein was observed in C2C12 cells transfected with Pc-hR plasmids (Figures $1(\mathrm{~B})$ and $1(\mathrm{C}-\mathrm{b})$ ), but was not observed in $\mathrm{C} 2 \mathrm{C} 12$ cells transfected with $\mathrm{Pc}-3.1$ plasmids (Figures $1(\mathrm{~B})$ and $1(\mathrm{C}-\mathrm{a})$ ). The expression of $\mathrm{hR}$ in COS7 cells was identified by Western blot (Figure 1(D)). HR 


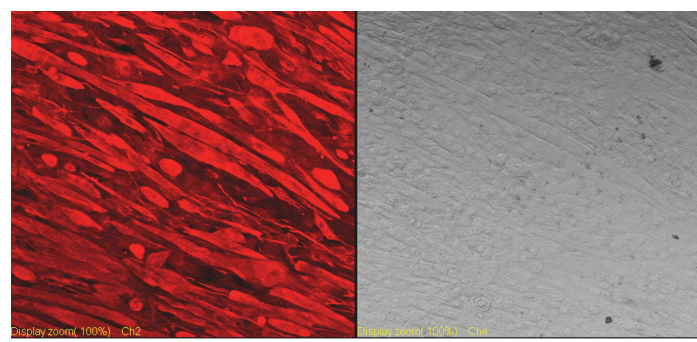

C2C12 transfected with Pc-3.1

(a)

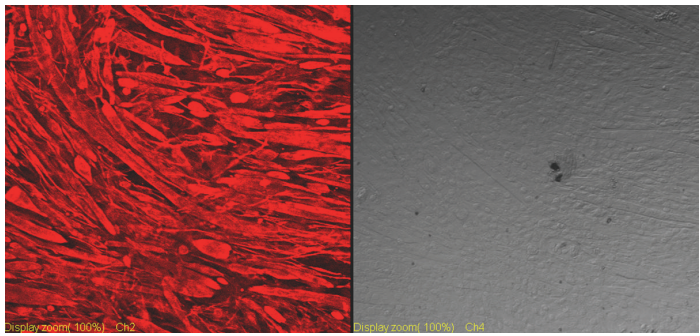

C2C12 transfected with Pc-3.1

(c)

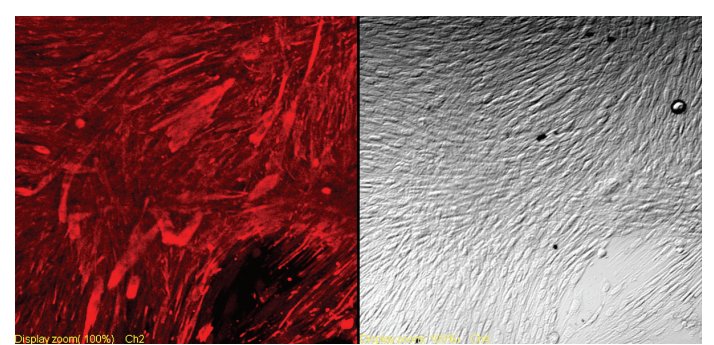

C2C12 transfected with Pc-hR

(b)

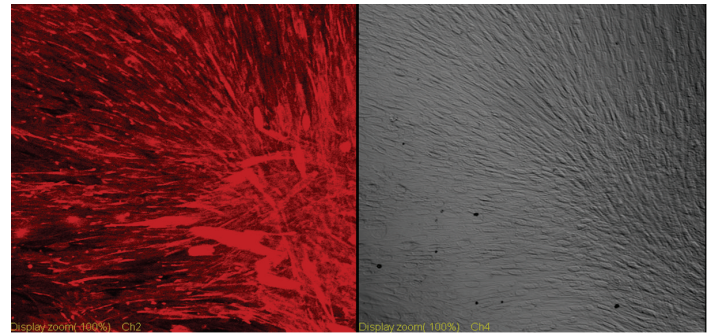

C2C12 transfected with Pc-hR

(d)

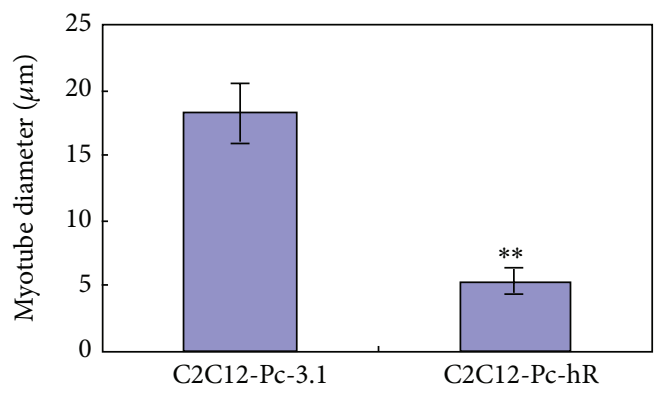

(e)

FIGURE 3: Effects of hR on myogenic differentiation of C2C12 cells (immunofluorescence staining). ((a) and (b)) The expression of desmin in C2C12 myotubes transfected with Pc-3.1 and transfected with Pc-hR vectors (magnification 200x). ((c) and (d)) The expression of myoglobin in C2C12 myotubes transfected with Pc-3.1 and transfected with Pc-hR vectors (magnification 200x). (e) Analysis of myotubes diameter. ${ }^{* *} P<0.01$.

expressed significantly in COS7 cells transfected with Pc-hR whereas not expressed in control cells.

\subsection{Effects of hR on Proliferation and Cell Cycle of C2C12 Cells.} MTT assays were performed to determine cell proliferation. Compared with the control, proliferation of $\mathrm{C} 2 \mathrm{C} 12$ cells transfected with Pc-hR was significantly promoted (Figure 2(a)). Overexpression of hR also significantly induced the $\mathrm{S}$ cycle accumulation in $\mathrm{C} 2 \mathrm{C} 12$ cells. There were $26.9 \%$ of the $\mathrm{S}$ phase cells in $\mathrm{C} 2 \mathrm{C} 12$ cells transfected with $\mathrm{Pc}-\mathrm{hR}$ versus $7.0 \%$ in the control groups $(P<0.01)$ (Figures 2(b), 2(c), and 2(d)). At the same time, overexpression of hR significantly reduced the ratio of $\mathrm{G} 0 / \mathrm{G} 1$ cycle in $\mathrm{C} 2 \mathrm{C} 12$ cells transfected with $\mathrm{Pc}-\mathrm{hR}$ $(61.1 \%$ versus $76.2 \%$ in the control) $(P<0.01)$ (Figures $2(\mathrm{~b})$, 2(c), and $2(\mathrm{e}))$.

3.3. Effects of hR on Myogenic Differentiation of C2C12 Cells. Desmin is a muscle-specific intermediate filament protein which is expressed in both smooth and striated muscles. It and myoglobin are important markers of myogenic differentiation. Immunofluorescence staining was performed to detect the expression of desmin and myoglobin. Compared with the control cells transfected with Pc-3.1, expression of desmin and myoglobin decreased significantly in Pc-hR-transfected C2C12 myotubes (Figures 3(a), 3(b), 3(c), and 3(d)). HR also decreased the diameters of myotubes $(P<0.01)$ (Figure $3(\mathrm{e}))$. These data suggest that $\mathrm{hR}$ inhibited myogenic differentiation of $\mathrm{C} 2 \mathrm{C} 12$ myoblasts.

3.4. Effects of hR on Glucose Uptake and Expression of Relevant Genes in C2C12 Myotubes. Data showed that insulinstimulated glucose uptake in $\mathrm{C} 2 \mathrm{C} 12$ myotubes transfected with Pc-hR was significantly decreased as compared with controls (Figure 4(a)). To further investigate the mechanisms, we examined the mRNA expression of IR and GLUT4 of C2C12 myotubes transfected with Pc-3.1 and Pc-hR plasmids. 


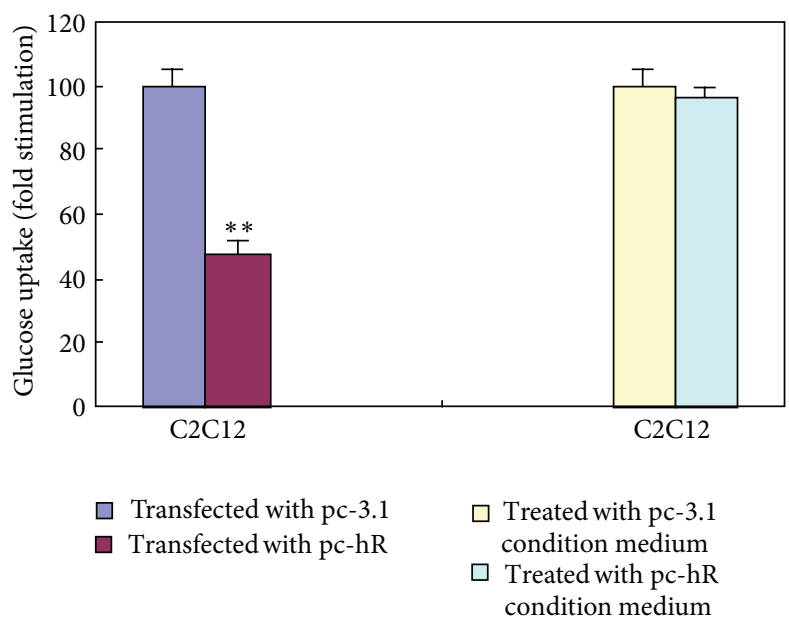

(a)
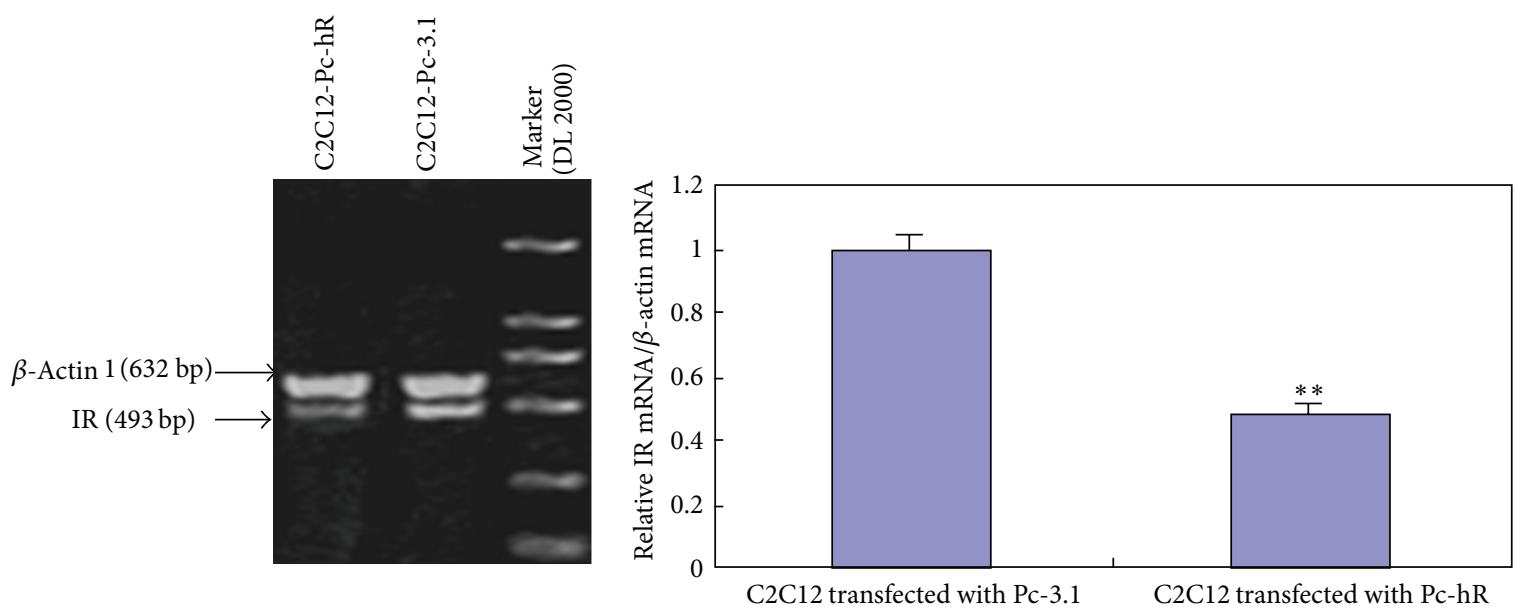

(b)
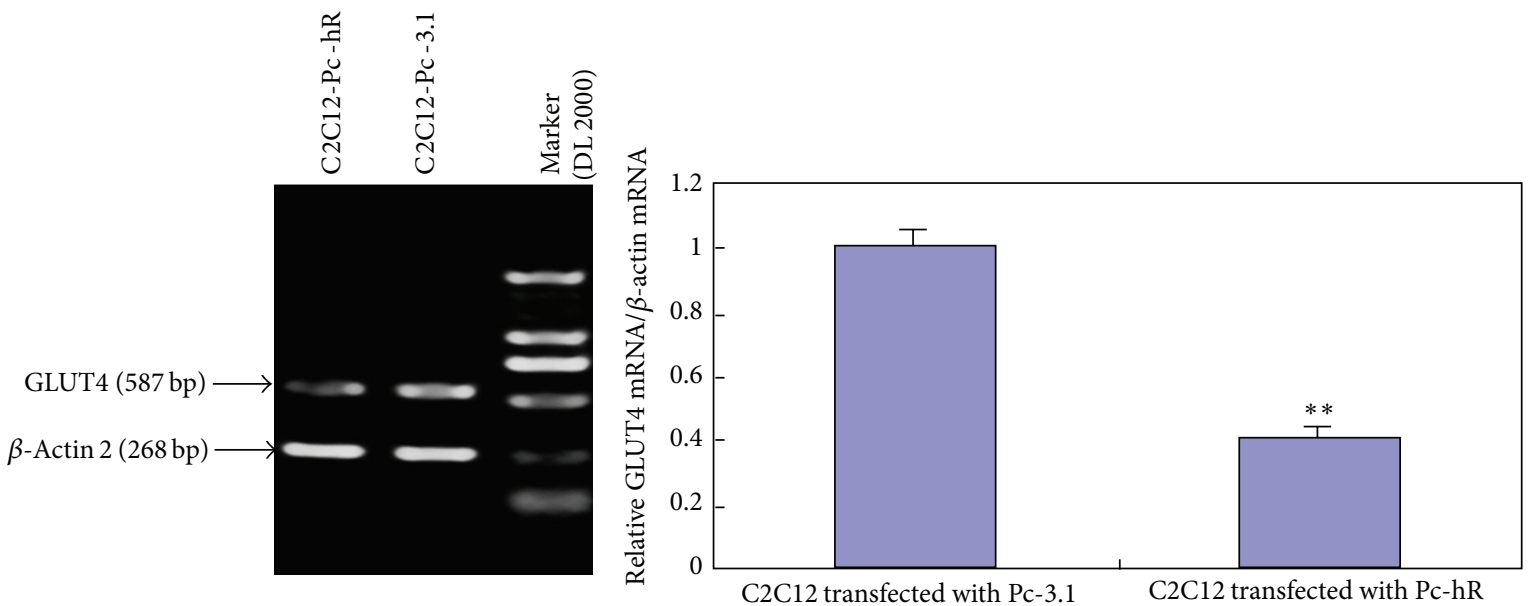

(c)

FIGURE 4: Effects of hR on glucose uptake and expression of relevant genes in C2C12 myotubes. (a) The effects of hR on glucose uptake in C2C12 myotubes. (b) Expression of insulin receptor (IR). (c) Expression of glucose transporter 4 (GLUT4). The amount of each target mRNA was normalized by the amount of $\beta$-actin mRNA and was expressed relative to the abundance of the target mRNA in cells transfected with Pc-3.1. ${ }^{* *} P<0.01$. 
Semiquantitative RT-PCR analysis showed that expression of IR and GLUT4 mRNA decreased significantly in C2C12 myotubes transfected with $\mathrm{Pc}-\mathrm{hR}$ as compared with the controls $(P<0.01)$ (Figures $4(\mathrm{~b})$ and $4(\mathrm{c}))$.

To observe whether $\mathrm{hR}$ has a direct effect on insulin sensitivity of myocytes, condition mediums of COS7 cells transfected with Pc-3.1 and Pc-hR were used to culture C2C12 myotubes. The result showed that there was no significant difference in glucose uptake between the myotubes treated with Pc-hR condition medium and the myotubes treated with the Pc-3.1 condition medium (Figure 4(a)).

\section{Discussion}

Since the discovery of resistin in 2001, there has been controversy on the role of resistin on glucose metabolism of skeletal muscles. It was reported not only that the resistin had no effect on insulin sensitivity of skeletal muscles $[2,20]$ but also that the resistin impaired insulin sensitivity of myocytes $[3,21,22]$. However, in the studies where resistin induced insulin resistance in myocytes, its mechanism was different or even contradictory. Fan et al. [3] and Palanivel et al. [21] thought that resistin impaired insulin sensitivity of rat skeletal muscle cells (differentiated from L6 myoblasts) by the inhibition of PI-3K insulin signal transduction pathway. Moon et al. [22] and his colleagues transfected recombined mouse resistin vector in L6 cells and the glucose uptake decreased significantly whereas PI-3K insulin signal transduction pathway was not affected. So they presumed that resistin degraded intrinsic activity of GLUTs. However, it was not mentioned whether resistin affects proliferation and differentiation of L6 myoblasts in these studies.

In the current study, we chose $\mathrm{C} 2 \mathrm{C} 12$ myotubes as a cell culture model to test whether resistin can induce insulin resistance in muscle cells. $\mathrm{C} 2 \mathrm{C} 12$ cells are immortalized cell lines from the $\mathrm{C} 3 \mathrm{H}$ mouse skeletal muscle satellite cells, often used as the model of the study of the development and differentiation of muscle lineage [23], and insulin resistance is readily induced in this cell model with palmitate [24]. In order to observe the effect of resistin on muscle cell differentiation for a long time, resistin gene was expressed in $\mathrm{C} 2 \mathrm{C} 12$ cells by the stable transfection technology so that it can offer the continuing effect of resistin on $\mathrm{C} 2 \mathrm{C} 12$ cells. As well as to observe the direct effect of resistin on $\mathrm{C} 2 \mathrm{C} 12$ myotubes, resistin gene was expressed in COS7 cells by the transient transfection technology so that it can offer the resistin protein direct effect on $\mathrm{C} 2 \mathrm{C} 12$ myotubes.

That resistin inhibits adipogenic differentiation has been reported $[5,12,14]$. However, to our knowledge, its effect on myogenic differentiation has not been reported, but it was reported that Fizzl, which also belongs to Fizz family inhibited myogenesis [25]. Our data showed that hR dramatically stimulated the proliferation and increase of $S$ phase cells whereas decreased G0/G1 phase cells. It also decreased diameter of myotubes and suppressed the expression of myogenic markers including desmin and myoglobin. These results indicate that resistin inhibits myogenesis and promotes proliferation of immature myocytes. Compared with the control cells, the glucose uptake and expression of IR and GLUT4 were dramatically decreased in $\mathrm{C} 2 \mathrm{C} 12$ myotubes. However, compared with the controls, hR condition medium did not affect glucose uptake of $\mathrm{C} 2 \mathrm{C} 12$ myotubes; nevertheless, it inhibits myogenesis and promotes proliferation of immature myocytes so that the population of mature myocytes and expression of IR and GLUT4 cutdown, which led to less glucose uptake.

In a word, our results demonstrated for the first time that human resistin acted on cell cycle of myoblasts, inhibited myogenic differentiation, and promoted proliferation of myoblasts, which may be relevant to the glucose metabolic disorder.

\section{Author's Contribution}

Dr Zhen Wu Du and Chun Hua Sheng contributed equally to this work that Dr Zhen Wu Du and Chun Hua Sheng is owned first author.

\section{Acknowledgments}

The authors thank Professor Pei-Yin Zhang and Professor Xin-Rui Wang (Jilin University) for their great helps in the assays of glucose uptake ratio and immunofluorescence staining and confocal microscopy. This study was supported in part by grants from the Scientific Research Foundation of Jilin province (nos. 2008-2123, 20110740, 20100942) and Grants from the Health Scientific Research Foundation of Jilin province (no. 2010z083). of Recombinant Human Resistin (hR) Expression Vector and Transfection into

\section{References}

[1] C. M. Steppan, S. T. Bailey, S. Bhat et al., "The hormone resistin links obesity to diabetes," Nature, vol. 409, no. 6818, pp. 307-312, 2001.

[2] M. W. Rajala, S. Obici, P. E. Scherer, and L. Rossetti, "Adiposederived resistin and gut-derived resistin-like molecule- $\beta$ selectively impair insulin action on glucose production," Journal of Clinical Investigation, vol. 111, no. 2, pp. 225-230, 2003.

[3] H. Q. Fan, N. Gu, F. Liu et al., "Prolonged exposure to resistin inhibits glucose uptake in rat skeletal muscles," Acta Pharmacologica Sinica, vol. 28, no. 3, pp. 410-416, 2007.

[4] E. D. Muse, T. K. T. Lam, P. E. Scherer, and L. Rossetti, "Hypothalamic resistin induces hepatic insulin resistance," Journal of Clinical Investigation, vol. 117, no. 6, pp. 1670-1678, 2007.

[5] K. H. Kim, L. Zhao, Y. Moon, C. Kang, and H. S. Sul, "Dominant inhibitory adipocyte-specific secretory factor (ADSF)/resistin enhances adipogenesis and improves insulin sensitivity," Proceedings of the National Academy of Sciences of the United States of America, vol. 101, no. 17, pp. 6780-6785, 2004.

[6] C. M. Steppan and M. A. Lazar, "The current biology of resistin,” Journal of Internal Medicine, vol. 255, no. 4, pp. 439-447, 2004.

[7] P. G. McTernan, F. M. Fisher, G. Valsamakis et al., "Resistin and type 2 diabetes: regulation of resistin expression by insulin and rosiglitazone and the effects of recombinant resistin on lipid and glucose metabolism in human differentiated adipocytes," 
Journal of Clinical Endocrinology and Metabolism, vol. 88, no. 12, pp. 6098-6106, 2003.

[8] M. S. Farvid, T. W. K. Ng, D. C. Chan, P. H. R. Barrett, and G. F. Watts, "Association of adiponectin and resistin with adipose tissue compartments, insulin resistance and dyslipidaemia," Diabetes, Obesity and Metabolism, vol. 7, no. 4, pp. 406-413, 2005.

[9] J. Janowska, B. Zahorska-Markiewicz, and M. OlszaneckaGlinianowicz, "Relationship between serum resistin concentration and proinflammatory cytokines in obese women with impaired and normal glucose tolerance," Metabolism, vol. 55, no. 11, pp. 1495-1499, 2006.

[10] I. Nagaev and U. Smith, "Insulin resistance and type 2 diabetes are not related to resistin expression in human fat cells or skeletal muscle," Biochemical and Biophysical Research Communications, vol. 285, no. 2, pp. 561-564, 2001.

[11] C. C. Zou, L. Liang, F. Hong, J. F. Fu, and Z. Y. Zhao, "Serum adiponectin, resistin levels and non-alcoholic fatty liver disease in obese children," Endocrine Journal, vol. 52, no. 5, pp. 519-524, 2005.

[12] K. H. Kim, K. Lee, Y. S. Moon, and H. S. Sul, "A cysteinericha diposet issue-specifics ecretory factor inhibits adipocyte diferentiation," The Journal of Biological Chemistry, vol. 276, pp. 11252-11256.

[13] R. Nogueiras, R. Gallego, O. Gualillo et al., "Resistin is expressed in different rat tissues and is regulated in a tissue- and genderspecific manner," FEBS Letters, vol. 548, no. 1-3, pp. 21-27, 2003.

[14] T. Ort, A. A. Arjona, J. R. MacDougall et al., "Recombinant human FIZZ3/resistin stimulates lipolysis in cultured human adipocytes, mouse adipose explants, and normal mice," Endocrinology, vol. 146, no. 5, pp. 2200-2209, 2005.

[15] S. D. Patel, M. W. Rajala, L. Rossetti, P. E. Scherer, and L. Shapiro, "Disulfide-dependent multimeric assembly of Resistin family hormones," Science, vol. 304, no. 5674, pp. 1154-1158, 2004.

[16] B. Aruna, S. Ghosh, A. K. Singh et al., "Human recombinant resistin protein displays a tendency to aggregate by forming intermolecular disulfide linkages," Biochemistry, vol. 42, no. 36, pp. 10554-10559, 2003.

[17] L. Hari, V. Brault, M. Kléber et al., "Lineage-specific requirements of $\beta$-catenin in neural crest development," Journal of Cell Biology, vol. 159, no. 5, pp. 867-880, 2002.

[18] Y. Nakamori, M. Emoto, N. Fukuda et al., "Myosin motor Myolc and its receptor NEMO/IKK- $\gamma$ promote TNF- $\alpha$-induced serine307 phosphorylation of IRS-1," Journal of Cell Biology, vol. 173, no. 5, pp. 665-671, 2006.

[19] C. M. Kusminski, N. F. Da Silva, S. J. Creely et al., “The in vitro effects of resistin on the innate immune signaling pathway in isolated human subcutaneous adipocytes," Journal of Clinical Endocrinology and Metabolism, vol. 92, no. 1, pp. 270-276, 2007.

[20] D. Dietze, M. Koenen, and K. Röhrig, "Impairment of insulin signaling in human skeletal muscle cells by co-culture with human adipocytes," Diabetes, vol. 51, no. 8, pp. 2369-2376, 2002.

[21] R. Palanivel, A. Maida, Y. Liu, and G. Sweeney, "Regulation of insulin signalling, glucose uptake and metabolism in rat skeletal muscle cells upon prolonged exposure to resistin," Diabetologia, vol. 49, no. 1, pp. 183-190, 2006.

[22] B. Moon, J. J. M. Kwan, N. Duddy, G. Sweeney, and N. Begum, "Resistin inhibits glucose uptake in L6 cells independently of changes in insulin signaling and GLUT4 translocation," American Journal of Physiology, vol. 285, no. 1, pp. E106-E115, 2003.
[23] T. Tamaki, A. Akatsuka, K. Ando et al., "Identification of myogenic-endothelial progenitor cells in the interstitial spaces of skeletal muscle," Journal of Cell Biology, vol. 157, no. 4, pp. 571-577, 2002.

[24] T. Coll, D. Álvarez-Guardia, E. Barroso et al., "Activation of peroxisome proliferator-activated receptor- $\delta$ by GW501516 prevents fatty acid-induced nuclear factor- $\kappa \mathrm{B}$ activation and insulin resistance in skeletal muscle cells," Endocrinology, vol. 151, no. 4, pp. 1560-1569, 2010.

[25] B. Blagoev, I. Kratchmarova, M. M. Nielsen et al., "Inhibition of adipocyte differentiation by resistin-like molecule $\alpha$ : biochemical characterization of its oligomeric nature," Journal of Biological Chemistry, vol. 277, no. 44, pp. 42011-42016, 2002. 


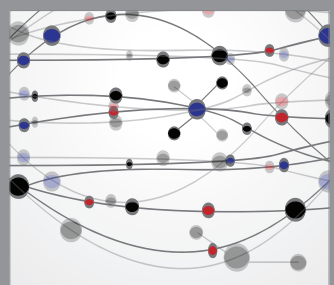

The Scientific World Journal
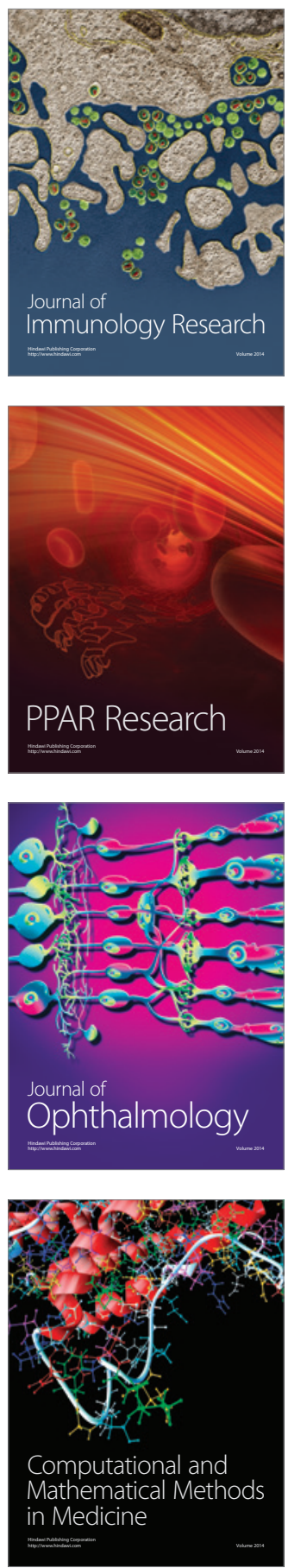

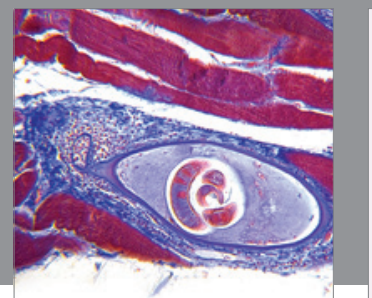

Gastroenterology

Research and Practice
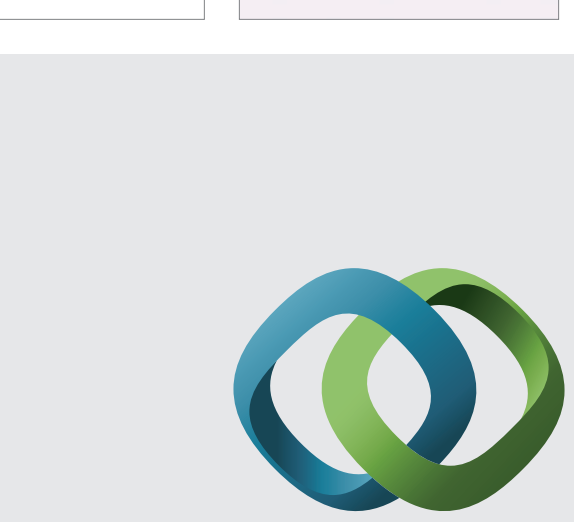

\section{Hindawi}

Submit your manuscripts at

http://www.hindawi.com
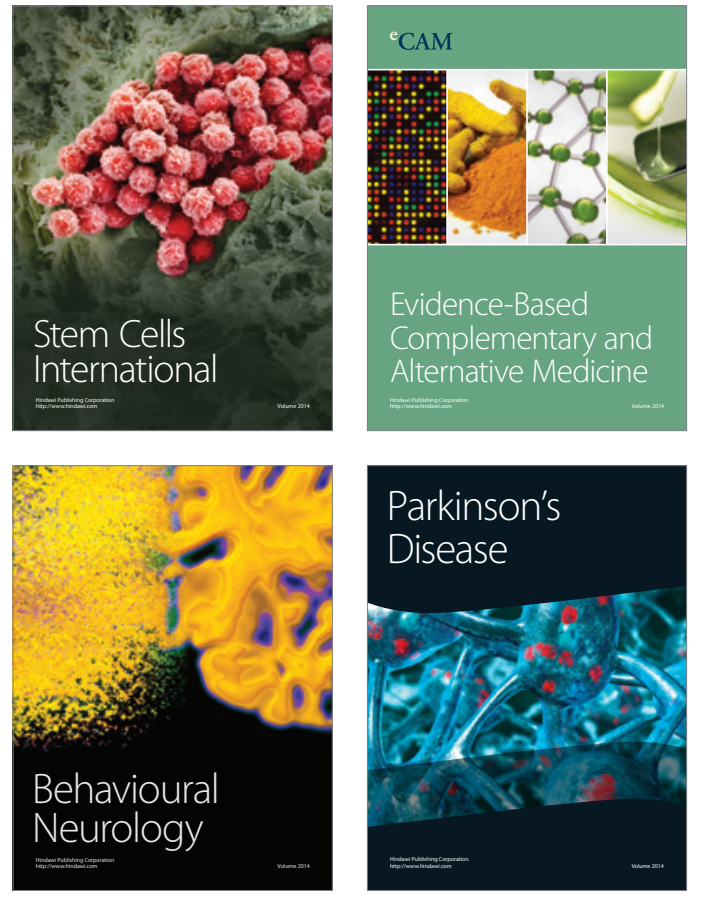
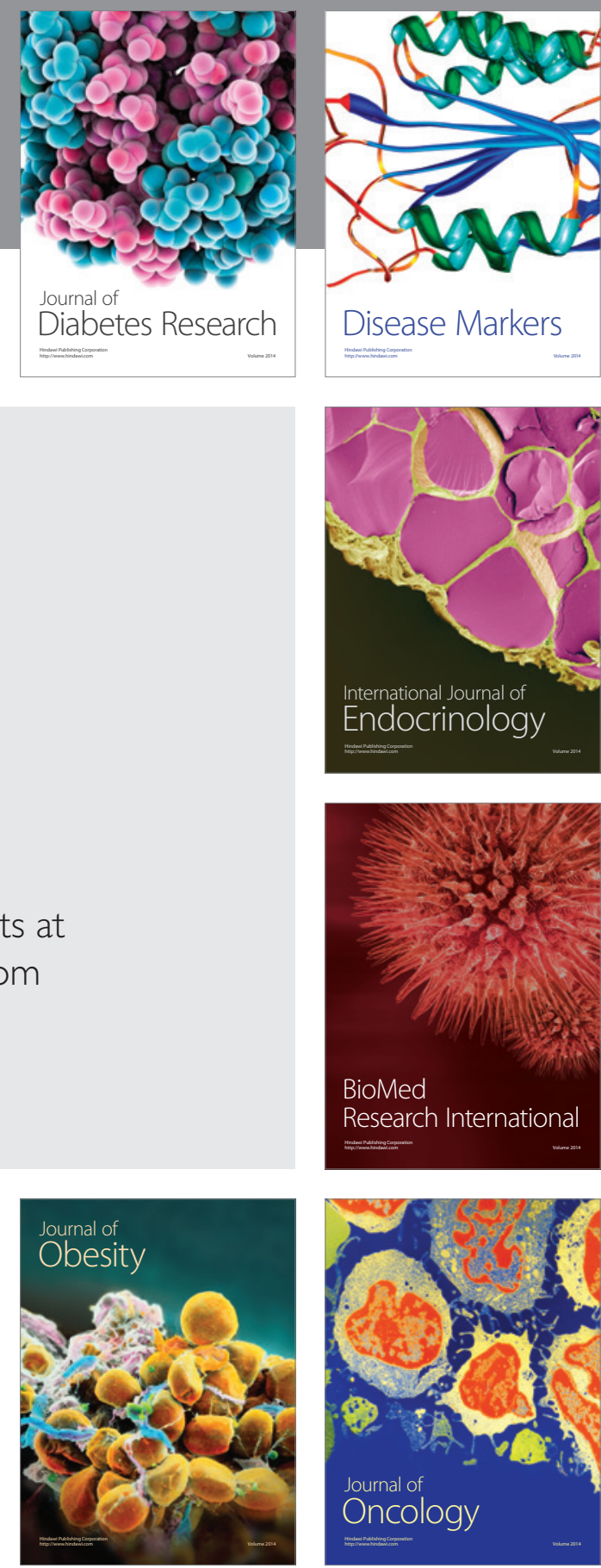

Disease Markers
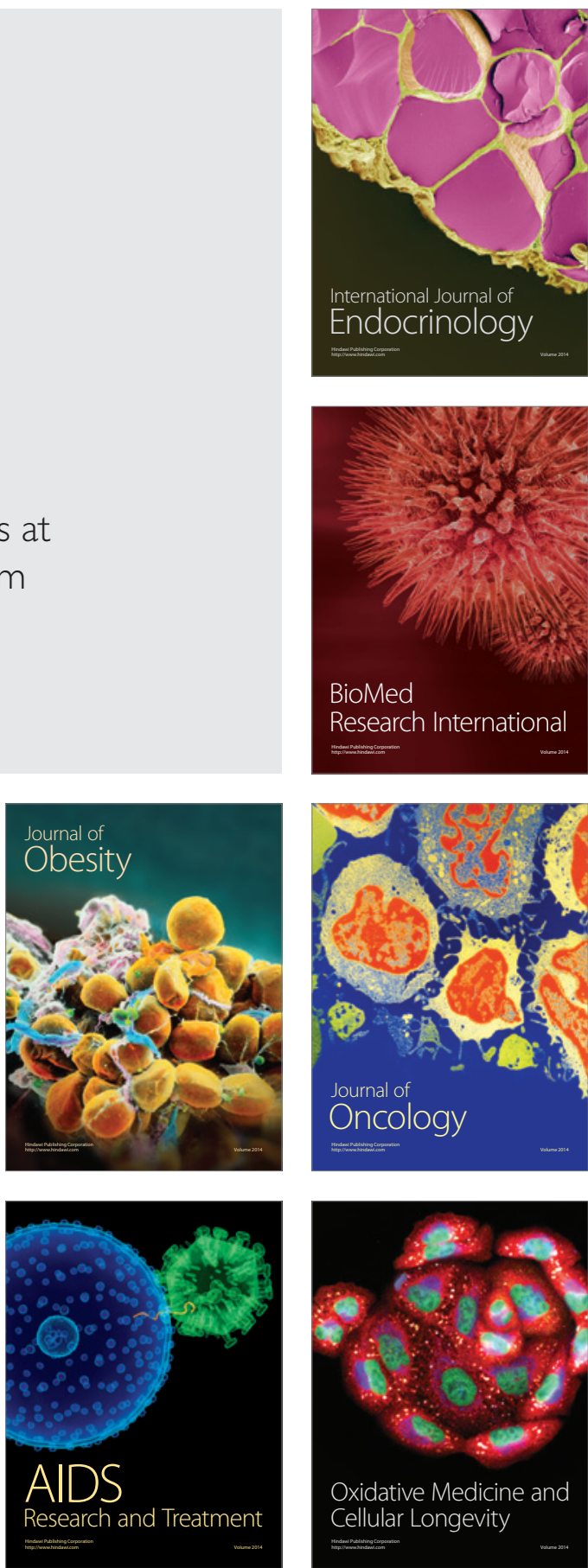